\title{
Variación de la velocidad del centro de presiones en deportistas con esguince lateral de tobillo
}

\author{
Variation of the Velocity of the Center of Pressure in Athletes With Lateral \\ Ankle Sprain
}

\author{
Miguel Blanco-Traba ${ }^{1,2,3,6}$, Manuel MosQueira-Ouréns ${ }^{1,4,5,6}$ \\ ${ }^{1}$ Diplomado en Podología \\ podo.mosqueira@gmail.com \\ ${ }^{2}$ Postgrado en Patomecánica del pie y sus tratamientos ortopodológicos; UB \\ ${ }^{3}$ Máster Oficial de Investigación en Podología; Universidad Rey Juan Carlos de Madrid \\ ${ }^{4}$ Máster en Investigación Sanitaria,especialista reeducación funcional; UDC \\ podologiamiguel@gmail.com \\ ${ }^{5}$ Doctorando en ciencias de la salud; UDC \\ ${ }^{6}$ Podólogo en consulta privada
}

Correspondencia:

Blanco Traba, Miguel

Teléfono: 663524661

Dirección Postal: Plaza Domingo A. Andrade $112^{\circ} \mathrm{B}$

E-15270 A Coruña

Correo electrónico: podologiamiguel@gmail.com

Fecha de recepción: 8 de septiembre de 2013

Fecha de aceptación: 2 de octubre de 2013

Los autores declaran no tener ningún tipo de interés económico o comercial.

\section{RESUMEN}

Introducción. La inestabilidad crónica de tobillo es un cuadro clínico muy frecuente e incapacitante en deportistas. No se conoce con certeza la etiología de la misma siendo propuestas las alteraciones biomecánicas y déficit de equilibrio postural, por deterioro en los sistemas involucrados en la propiocepción. En este estudio se valoran las consecuencias biomecánicas en personas que padecieron esguince lateral de tobillo.

Material y métodos. Se han analizado los datos baropodométricos de 60 personas que practican deporte habitualmente (30 grupo esguinces, 30 grupo control) entre 18 y 40 años.

Se estudió la velocidad del CoP siguiendo las indicaciones del software de la plataforma FootScan ${ }^{\circledR}$. Los datos han sido recopilados in situ, por 2 clínicos en diferente tiempo y lugar.

Resultados. Se han encontrado diferencias estadísticamente significativas (0,031 para un IC del 95\%) en los valores de: inicio de antepié plano izquierdo y rocker de talón izquierdo.

No se han encontrado diferencias entre hombres y mujeres.

Conclusiones. Se han encontrado muy pocas diferencias entre el grupo esguince y el grupo control por lo que nuestra hipótesis se ha rechazado, no habiendo diferencias significativas en la biomecánica de la marcha de deportistas que han padecido esguince lateral de tobillo comparados con personas sanas. Futuros estudios son necesarios para conocer con mayor profundidad los factores de alteración biomecánica tras un esguince de tobillo, analizando diferentes factores biomecánicos, podobarométricos y diferencias de género.

Palabras clave: lesiones del tobillo; esguince tobillo; inestabilidad de tobillo; inestabilidad crónica de tobillo.

\section{ABSTRACT}

Introduction. The chronic ankle instability is a very common clinical profile that incapacitates athletes. It is not known with certainty the etiology of it being proposed biomechanical alterations and postural balance deficit, impairment of the systems involved in propioception. This study evaluated the biomechanical consequences in people who suffered lateral ankle sprain.

Material and methods. Baropodometics data of 60 people who practiced sports regularly ( 30 strains group, 30 control group) between 18 and 40 have been analyzed. 
We studied the speed of CoP following the indications of the software FootScan ${ }^{\circledR}$ platform. The data has been collected in situ by 2 clinics in different time and place.

Results. There were statistically significant differences (0.031 with IC 95\%) in values: left flat forefoot and left heel rocker.

No differences were found between men and women.

Conclusions. There were found a low amount of differences between the sprain group and the control group so our hypothesis was rejected, with no significant differences in gait biomechanics of athletes who have experienced lateral ankle sprain compared with healthy people. Future studies are needed to know biomechanical alteration factors after an ankle sprain, analyzing different biomechanical and podobarometric factors and gender differences.

Key words: ankle foot injuries; ankle sprain; ankle instability; chronic ankle instability.

Sumario: 1. Introducción. 2. Material y métodos. 3. Resultados. 4. Discusión. 5. Conclusiones. Bibliografía. Anexos.

Referencia normalizada: Blanco-Traba, M., Mosquera-Ouréns, M. Variación de la velocidad del centro de presiones en deportistas con esguince lateral de tobillo. Rev. Int. Cienc. Podol. 2014; 8(2): 119-131. 


\section{INTRODUCCIÓN}

El esguince lateral de tobillo es una lesión que padecen entre el $20 \%$ y el $40 \%$ de atletas y una de las lesiones más frecuentes ${ }^{1}, 14 \%$ del total de lesiones deportivas, del cual $77 \%$ son esguinces lateral externos, siendo las actividades de carrera (25\%), deportes con raqueta $(20 \%)$, juegos de pelota (19\%) y fútbol (14\%) los que mayor riesgo presentan ${ }^{2}$. El esguince lateral de tobillo se produce por un movimiento brusco en inversión, cuando el centro de gravedad del atleta se desplaza sobre el borde lateral de la pierna en carga, haciendo que el tobillo ruede hacia dentro a una alta velocidad. Cuando hay daño en el control postural, se producen variaciones en el centro de presiones, no solamente en el tobillo lesionado sino también en el contra-lateral no lesionada, lo que sugiere trastornos mediados a nivel central ${ }^{10}$. Otras estructuras asociadas verse afectadas durante el esguince, como los tendones, cápsula articular y terminaciones nerviosas propioceptivas ${ }^{1}$, que provocan un déficit propioceptivo y biomecánico del tobillo y pie $^{3}$. Los mecano-receptores y receptores sensoriales como el órgano tendinoso de Golgi, proporcionan información en forma de impulsos aferentes asociados a la tensión de tendones, movimiento articular y posición así como contribuyen al sistema reflejo complejo, que actúan manteniendo el equilibrio del cuerpo. La cronificación de la lesión produce desequilibrios que pueden persistir durante años ${ }^{11}$. Después de un esguince lateral externo, los síntomas agudos suelen mejorar en pocas semanas, pero algunos deportistas continúan con dolor, sensación de inestabilidad y edema crónico ${ }^{4}$. La inestabilidad crónica de tobillo se estima que afecta al 10-30\% de lesiones agudas y es pobremente entendido por investigadores y clínicos, dando lugar a incoherencias en terminología, definiciones e hipótesis y resultados contradictorios en investigación ${ }^{2,4,5}$. Se estima que los problemas residuales pueden persistir durante décadas, y muchos deportistas, no pueden volver a su nivel anterior de actividad por persistir sus síntomas ${ }^{2,6,7,8}$. El desarrollo del deterioro y limitación de la actividad es independiente de la severidad de la lesión inicial, y no se limita a la extremidad lesionada. Se han asociado problemas de inestabilidad crónica de tobillo, en el pie contra-lateral, en el $85 \%$ de personas, después de un esguince unilateral ${ }^{9}$.

Existen varias teorías para dilucidar por qué se produce una inestabilidad crónica. Una de ellas fue el déficit de fuerza de la musculatura eversora, pero la evidencia ha concluido que no es un factor significativo en el desarrollo de problemas funcionales, siendo descartada ${ }^{11,12}$, además al caminar, los peroneos se activan con más fuerza para proteger el tobillo lesionado y esto puede provocar debilidad excéntrica de la musculatura inversora ${ }^{11}$ que al mismo tiempo es inhibida selectivamente por su capacidad de iniciar el movimiento en dirección hacia la zona lesionada ${ }^{11,12,13}$.

Otros autores sugieren, como factor causante de esguinces de repetición, una inversión brusca y desproporcionada del pie como compensación a una posición de máxima pronación por déficit postural de la musculatura inversora. En esta línea Munn et al proponen que cuando el cuerpo se balancea lateralmente, en carga, más allá del rango de eversión de la subastragalina (ASA), este lleva a cabo una inversión brusca, con el consecuente momento inversor liberando la articulación de la ASA que se encontraba en máxima pronación, por ello para evitar el daño articular, se produce una contracción e inversión repentina ${ }^{7,12}$, esta idea soporta que el déficit de fuerza excéntrica de la musculatura inversora debería contribuir a síntomas de inestabilidad de tobillo debido a una reducción de la capacidad para controlar la oscilación postural lateral en carga ${ }^{7,14}$. En la misma línea, sobre la eversión del pie como causante, T Willems et al en su estudio prospectivo sobre esguinces por inversión y comparándolo con un grupo control han encontrado varios hallazgos significativos de que, el grupo de esguinces de tobillo, tiene el pie más evertido que el grupo control. Otros autores han estudiado la posición del tobillo y las presiones plantares durante la dinámica concluyendo que en la fase de contacto inicial de talón se produce una mayor inversión en sujetos con inestabilidad de tobillo ${ }^{11}$. En otros estudios, la inversión se produce justo antes del choque de talón y después del despegue de este, con un incremento en la electromiografía del peroneo lateral largo después de elevar el talón ${ }^{7,15}$. Más autores analizaron las presiones plantares donde sugieren que el 
grupo con Inestabilidad crónica tiene mayores presiones plantares laterales y ello es un factor que predispone a la inestabilidad lateral ${ }^{16,17,18}$, sin embargo, en un estudio de Huang PY, vieron un cambio del cop de lateral a medial más rápido en pacientes con inestabilidad así como un mayor pico de presión en M1, que suelen ser indicadores de pronación del pie por colapso del arco longitudinal interno. Roel De Ridder, contradice esta distribución de presiones más lateral ${ }^{16,17,18}$ en pacientes con Inestabilidad crónica ${ }^{19}$ y T. Willems et al hallaron mayor presión bajo la zona medial del pie, un retraso en la flexión de rodilla máxima, una resupinación retrasada en la fase de propulsión, un despegue por zona lateral y no por el hallux, un tiempo total de apoyo del pie más largo que el grupo control, un mayor rango de movimiento en $1^{\mathrm{a}}$ metatarsofalángica ${ }^{20}$, todo ello sugiere un aumento de momentos pronadores. Los factores de riesgo anatómicos que se encuentran asociados a la aparición de un esguince lateral, son principalmente el rango de eversión calcánea en mujeres y el incremento de inclinación del astrágalo en hombres ${ }^{20}$. Los resultados sobre la inestabilidad crónica de tobillo son débiles, aunque si hay consenso en afirmar que la causa es multifactorial: alteración a nivel central, alteración postural/propioceptivo, retraso en la activación de la musculatura eversora y debilidad en musculatura inversora son algunas de ellas ${ }^{11,12,13,14}$.

En este estudio no se pretende valorar las causas de la inestabilidad crónica de tobillo a causa de un esguince sino en las consecuencias que este provoca en el pie y su biomecánica.

Hay evidencia de una debilidad excéntrica de la musculatura inversora, también se ha hallado un aumento de la activación de peroneos para proteger la zona lesionada, mayor inversión en choque de talón, con una posible compensación en pronación a nivel de la articulación subta$\operatorname{lar}^{18}$, mayor presión bajo M1 ${ }^{20}$, un aumento del tiempo de apoyo total del pie comparado con grupo control, fase de despegue con presión más lateral, mayor rango de movimiento de la $1^{\mathrm{a}}$ metatarsofalángica y alteraciones a nivel propioceptivo; todo ello nos hizo plantear la hipótesis de que en pacientes con inestabilidad crónica de tobillo existe un aumento de los momentos pronadores y una alteración de la biomecánica normal de la marcha.

\section{MATERIAL Y MÉTODOS}

Se ha realizado un estudio observacional analítico transversal con grupo control compuesto por deportistas no profesionales que realizan más de 8 horas de ejercicio a la semana.

Todos los participantes fueron evaluados a lo largo de un periodo de 8 semanas.

Para la elaboración de este estudio se ha realizado un muestreo no probabilístico por conveniencia.

La muestra elegida se ha realizado visitando a los deportistas en el lugar donde practican deporte que constó de 60 sujetos sanos de los cuales 30 padecieron esguince $(27$ hombres 3 mujeres; Edad: 28,50 \pm 5,507 años; Altura: $175,17 \pm 7,511$ centímetros; Peso: 74,67 \pm 9,293 kilogramos) y 30 (26 hombres 4 mujeres; Edad: 29,92 \pm 7,095 años; Altura: $176 \pm 5,837$ centímetros; Peso: 72,60 \pm 8,548 kilogramos) como grupo control.

Todos los participantes firmaron un consentimiento informado conforme a la ley orgánica de protección de datos 15/1999, de 13 de diciembre, de protección de datos de carácter personal.

Todo el estudio se realizó acorde a la normativa de la declaración de Helsinki. (Anexo 1 y 2).

\section{Criterios de inclusión}

Se han seleccionado los sujetos que cumplieran los siguientes criterios:

Deportista en activo, entre 18 y 40 años de edad que practicaran y practiquen deporte durante más de 8 horas a la semana y que no haya tenido ninguna lesión del MMII en los últimos 6 meses a mayores del esguince lateral externo.

Los esguinces deben haber ocurrido con más de 6 semanas de antigüedad y menos de 2 años para cumplir el diagnóstico de inestabilidad crónica de tobillo según las indicaciones de los autores $\mathbf{s}^{1,7,10,11,24,25}$.

\section{Criterios de exclusión}

Lesión aguda o crónica que afecte al equilibrio, lesiones vestibulares o neurológicas, hiperlaxi- 
tud, lesión en MMII, columna o sistema nervioso central, además de fracturas ${ }^{24,26}$.

También descartamos deformidades en pies como pie plano, hallux valgus, pie cavo o aducto $^{15}$.

\section{Material}

Para obtener los datos se ha utilizado la plataforma de presiones Footscan ${ }^{\circledR}$ Software Clinical (RSscan International, Belgium) 4096 sensores a $300 \mathrm{~Hz}$. La superficie de sensores de la plataforma es de 0,5 metros con 4 sensores/ $\mathrm{cm} 2$. Presenta marcado CE de acuerdo a la normativa vigente.

Los datos han sido obtenidos por 2 investigadores con experiencia.

Se realizaron 3 intentos de prueba ${ }^{27}$ obteniéndose las medidas mediante el método de 3 $\operatorname{pasos}^{28}$ y capturando 3 pisadas de cada pie para posteriormente obtener la media para fortalecer la fiabilidad de los resultados.

Se ha utilizado la velocidad del centro de presiones como medida de estudio ${ }^{21,22,23}$.

Conforme al software clínico de la Footscan ${ }^{\circledR}$ (RSscan International, Belgium) $^{19,20,22,27,29,30}$, la fase de apoyo puede ser dividida en 4 fases:

La primera, fase de contacto inicial, la cual es definida como el periodo desde el primer contacto del pie hasta el primer contacto de un metatarsiano.

La segunda fase es la fase de contacto del antepié, el cual es el periodo inmediatamente seguido a la fase de contacto inicial, hasta que el área de todas las cabezas metatarsales están en contacto con la plataforma.

La tercera fase es la de pie plano. Sigue al contacto de antepié y termina cuando el talón se levanta del suelo.

La cuarta fase es la de despegue del antepié, la cual empieza cuando el talón se eleva del suelo y termina cuando el pie deja de contactar con el suelo.

\section{Variables}

- Esguinces: Si o No. Es la variable dependiente, cualitativa dicotómica que utilizamos para comparar el grupo control con el grupo de personas con esguince.

- Variables socio-demográficas: La edad es una variable cuantitativa discreta que limitamos entre 18 y 40 años. El sexo es una variable cualitativa dicotómica dándole valores 1 para hombre y 0 para mujer. Se han utilizado para valorar si hay diferencias entre sexos.

- Variables antropométricas: Altura, peso, IMC. Variables cuantitativas continuas. La altura se mide en centímetros, el peso en kilos y el IMC en fracción de porcentaje con 3 decimales.

- Variables podobarométricas: variable cualitativa continua que surge de la media de 3 pisadas. Se miden en fracción de porcentaje con 3 decimales y se obtienen medidas de cada fase de la marcha para cada pie.

Primer contacto del pie (siempre es en el $0 \%$ por lo tanto no incluido como variable)

Fase de contacto de antepié: porcentaje en el que ocurre esta fase de la marcha (obtenida del programa informático Footscan ${ }^{\circledR}$ )

Fase de antepié plano: porcentaje en el que ocurre esta fase de la marcha (obtenida del programa informático Footscan ${ }^{\mathbb{}}$ )

Fase de elevación de talón: porcentaje en el que ocurre esta fase de la marcha (obtenida del programa informático Footscan ${ }^{\circledR}$ )

Ultimo contacto del pie (Siempre es el 100\% por lo tanto no incluida como variable)

Se han añadido los 3 rockers de la marcha calculados de la siguiente manera:

$1^{\text {er }}$ rocker: talón: Coincide con la fase de apoyo total de antepié obtenido por la plataforma de presiones. Ocurre desde el choque de talón hasta el contacto total del antepié momento en el cual el talón contacta totalmente con el suelo.

$2^{\circ}$ rocker: tobillo: Se calculó la diferencia desde el contacto total del antepié hasta la fase de elevación del talón. Este rocker ocurre desde que el pie está en fase plantígrada hasta que se eleva el talón por avance del miembro inferior contralateral.

$3^{\text {er }}$ rocker: metatarsal: Se calculó este rocker desde la fase de elevación del talón hasta la de despegue de los dedos siguiendo el $\%$ de la fase de la marcha que obtuvimos del footscan.

\section{Análisis estadístico de los datos}

Las variables cualitativas se mostraron con su distribución de frecuencias. Las variables cuantitativas se resumieron para su mejor comprensión a través de la media, su rango y su 


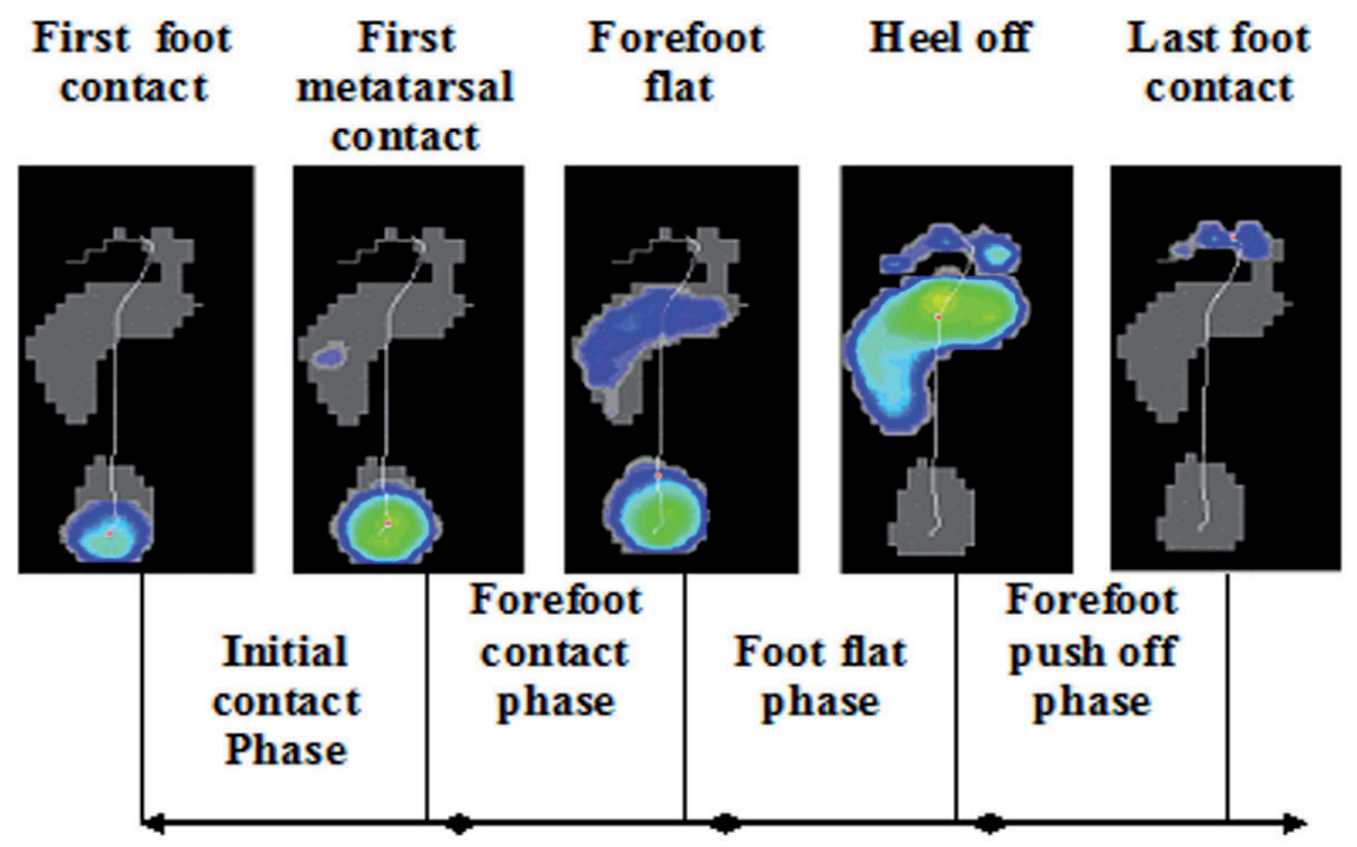

Figura 1. En esta imagen se muestra la captura baropodométrica de las fases de la marcha según el software Footscan ${ }^{\circledR}$. Los porcentajes de cada fase son obtenidos directamente por el programa informático ${ }^{19,20,22,27,29,30}$.

desviación estándar (DE) manejando un intervalo de confianza del $95 \%$ a lo largo de todo el desarrollo.

Para la comparación previa de los dos grupos nos valimos del test de la t-student para muestras independientes cuantitativas y para las variables cualitativas usamos el test chi cuadradro. Los resultados se mostraron en forma de diferencia de medias y proporciones con un IC de nuevo del 95\%.

En todos los casos comprobamos la distribución de la variable frente a los modelos teóricos y contrastábamos la hipótesis de homogeneidad de las varianzas.
Para procesar los datos se ha utilizado el programa estadístico SPPS versión 20.

\section{RESULTADOS}

Se ha calculado la media y la desviación típica de la edad, altura y peso de la muestra, tanto del grupo esguinces como el grupo control (Tabla 1).

Hemos realizado el test de chi-cuadrado para contrastar el grupo con esguince lateral externo de tobillo según el sexo no encontrándose diferencias significativas entre ambos sexos (Tablas 2 y 3 ).

\begin{tabular}{cccccc} 
& Esguince & N & Media & Desviación típ. & Error típ. de la media \\
\multirow{3}{*}{ Edad } & No & 30 & 29,93 & 7,095 & 1,295 \\
& $\mathrm{Si}$ & 30 & 28,50 & 5,507 & 1,005 \\
\multirow{3}{*}{ Altura } & $\mathrm{No}$ & 30 & 176,00 & 5,837 & 1,066 \\
& $\mathrm{Si}$ & 30 & 175,17 & 7,511 & 1,371 \\
& $\mathrm{No}$ & 30 & 72,60 & 8,548 & 1,561 \\
& $\mathrm{Si}$ & 30 & 74,67 & 9,293 & 1,697 \\
\hline
\end{tabular}

Tabla 1. Estadística descriptiva. 
Para medir la relación entre el grupo esguinces y el grupo control se ha utilizado la prueba t de student (Tabla 4).

Se han encontrado diferencias estadísticamente significativas en la fase de Inicio Antepie Plano Izquierdo y Rocker de talón izquierdo de 0,031 para un nivel de confianza del 95\%.

En las demás fases de la marcha y rockers no se han encontrado diferencias significativas entre el grupo control y el grupo de los esguinces.

ICMD/I: Inicio contacto metatarsiano derecho/izquierdo. IAPD/I: Inicio antepié plano derecho/izquierdo. ETD/I: Elevación talón derecho/izquierdo. RTaD/I: Rocker talón derecho/izquierdo. RtoD/I: Rocker tobillo derecho/ izquierdo. RMD/I: Rocker metatarsal derecho/ izquierdo.

\section{DISCUSIÓN}

Hemos comparado la diferencia entre hombres y mujeres sin obtener resultados concluyentes, esto podría deberse a la pequeña muestra de mujeres (4 grupo control y 3 grupo esguinces)

En el análisis comparativo de las fases de la marcha de los dos grupos, solamente se han encontrado diferencias en el inicio del antepié plano del pie izquierdo y en el rocker de talón del pie izquierdo. Esta diferencia puede ser debida a la posición más invertida del pie a la hora del choque de talón ${ }^{18}$ en el grupo de esguinces con respecto al grupo control como varios autores indican.

En el estudio de T. Willems et al $(\mathrm{N}=22)$, hallaron un retraso de la resupinación en la fase de propulsión y un tiempo total de apoyo del pie más largo que el grupo control ${ }^{20}$, en nuestro estudio las diferencias en estas fases no son significativas.

Se necesita realizar más investigación, en futuros estudios sería importante que en el grupo de esguinces estén diagnosticados como inestabilidad crónica de tobillo a través de formularios o pruebas objetivas para diferenciarlos del grupo control ya que si no presentan inestabilidad crónica los resultados pueden no ser estadísticamente significativos. Se sugiere un estudio prospectivo analizando antes del esguince de tobillo y varios meses después del mismo.

Los resultados obtenidos pueden servir como referencia para futuros estudios.

\section{CONCLUSIONES}

El propósito de este estudio ha sido valorar si hay alteraciones en la biomecánica de la marcha en personas deportistas que han padecido un esguince lateral de tobillo debido a un des-

\begin{tabular}{lcc} 
& Valor & Sig. asintótica (bilateral) \\
Chi-cuadrado de Pearson & $\mathbf{, 1 6 2}$ &, 688 \\
$\mathrm{~N}$ de casos válidos & 60 & \\
\hline
\end{tabular}

Tabla 2. Prueba de Chi-cuadrado.

\begin{tabular}{lccc} 
& & \multicolumn{2}{c}{ Valor } \\
& & Inferior & Superior \\
Razón de las ventajas para Sexo (Mujer / Hombre) & 1,385 &, 282 & 6,796 \\
Para la cohorte Esguince $=$ No & 1,165 &, 580 & 2,340 \\
Para la cohorte Esguince $=\mathrm{Si}$ &, 841 &, 344 & 2,059 \\
$\mathrm{~N}$ de casos válidos & 60 & & \\
\hline
\end{tabular}

Tabla 3. Prueba de estimación de riesgo. 


\begin{tabular}{|c|c|c|c|c|c|}
\hline & & & & & $\begin{array}{c}\text { Prueba T de igualdad de } \\
\text { medias } \\
\text { (IC 95\%) }\end{array}$ \\
\hline & Esguince & $\mathbf{N}$ & Media & Desviación típ. & Sig. (Bilateral) \\
\hline \multirow{2}{*}{ ICMD } & No & 30 & 0,10470 & 0,19682 & \multirow{2}{*}{0,638} \\
\hline & $\mathrm{Si}$ & 30 & 0,10107 & 0,37124 & \\
\hline \multirow{2}{*}{ ICMI } & No & 30 & 0,10880 & 0,18496 & \multirow{2}{*}{0,376} \\
\hline & $\mathrm{Si}$ & 30 & 0,10343 & 0,27268 & \\
\hline \multirow{2}{*}{ IAPD } & No & 30 & 0,18810 & 0,26620 & \multirow{2}{*}{0,254} \\
\hline & $\mathrm{Si}$ & 30 & 0,20287 & 0,064889 & \\
\hline \multirow{2}{*}{ IAPI } & No & 30 & 0,18267 & 0,24021 & \multirow{2}{*}{$\mathbf{0 , 0 3 1}$} \\
\hline & $\mathrm{Si}$ & 30 & 0,20160 & 0,40333 & \\
\hline \multirow[b]{2}{*}{ ETD } & No & 30 & 0,59473 & 0,94807 & \multirow[b]{2}{*}{0,836} \\
\hline & $\mathrm{Si}$ & 30 & 0,59967 & 0,89055 & \\
\hline \multirow{2}{*}{ ETI } & No & 30 & 0,57847 & 0,84971 & \multirow{2}{*}{0,117} \\
\hline & $\mathrm{Si}$ & 30 & 0,61370 & 0,86701 & \\
\hline \multirow{2}{*}{$\mathrm{RTaD}$} & No & 30 & 0,18810 & 0,026620 & \multirow{2}{*}{0,254} \\
\hline & $\mathrm{Si}$ & 30 & 0,20287 & 0,064889 & \\
\hline \multirow[b]{2}{*}{ RTaI } & No & 30 & 0,18267 & 0,024021 & \multirow{2}{*}{0,031} \\
\hline & $\mathrm{Si}$ & 30 & 0,20160 & 0,040333 & \\
\hline \multirow{2}{*}{ RToD } & No & 30 & 0,40663 & 0,94397 & \multirow{2}{*}{0,681} \\
\hline & $\mathrm{Si}$ & 30 & 0,39680 & 0,090117 & \\
\hline \multirow{2}{*}{ RToI } & No & 30 & 0,39580 & 0,084314 & \multirow{2}{*}{0,491} \\
\hline & $\mathrm{Si}$ & 30 & 0,41210 & 0,097483 & \\
\hline \multirow[b]{2}{*}{ RMD } & No & 30 & 0,40360 & 0,95611 & \multirow[b]{2}{*}{0,892} \\
\hline & $\mathrm{Si}$ & 30 & 0,40033 & 0,089055 & \\
\hline \multirow[b]{2}{*}{ RMI } & No & 30 & 0,42087 & 0,087417 & \multirow[b]{2}{*}{0,199} \\
\hline & $\mathrm{Si}$ & 30 & 0,39097 & 0,090748 & \\
\hline
\end{tabular}

Tabla 4. Significación de la prueba T. Media y Desviación típica de las variables. 
equilibrio muscular y postural. Los resultados obtenidos solamente muestran diferencias en la fase de inicio antepié plano izquierdo y rocker de tobillo izquierdo pero no en las demás fases de la marcha por lo que se necesita más investigación para poder afirmar que hay di- ferencias en la marcha entre las personas que han padecido esguinces y las que no.

\section{CONFLICTO DE INTERÉS}

No se ha producido ningún conflicto de interés.

\section{BIBLIOGRAFÍA}

1. Joshua C. Dubin, Doug Comeau, Rebecca I. McClelland, Rachel A. Dubin, Ernest Ferrel. Lateral and syndesmotic ankle sprain injuries: a narrative literature review. J Chiropr Med. 2011 Sep;10(3):204-19. http://dx.doi.org/10.1016/j.jcm.2011.02.001

2. Daniel TP Fong, Yue-Yan Chan, Kam-Ming Mok, Patrick SH Yung, Kai-Ming Chan. Understanding acute ankle ligamentous sprain injury in sports. Sports Med Arthrosc Rehabil Ther Technol. 2009 Jul 30;1:14. doi: 10.1186/1758-2555-1-14

3. Palmieri-Smith R, Hopkins JT, Brown TN. Peroneal activation deficits in persons with functional ankle instability. Am J Sports Med. 2009;37(5):982-8. doi: 10.1177/0363546508330147

4. Claire E. Hiller, Sharon L. Kilbreath, Kathryn M. Refshauge. Chronic Ankle Instability: Evolution of the Model. J Athl Train. 2011 Mar-Apr;46(2):133-41. doi: 10.4085/1062-6050-46.2.133

5. Anandacoomarasamy A, Barnsley L. Long term outcomes of inversion ankle injuries. Br J Sports Med. 2005;39:1-4.

6. Lofvenberg R, Karrholm J, Lund B. The outcome of nonoperated patients with chronic lateral instability of the ankle: a 20-year follow-up study. Foot Ankle Int. 1994;15(4):165-169.

7. Munn J, Beard D, Refshauge K, et al. Eccentric muscle strength in functional ankle instability. Med Sci Sports Exerc. 2003;35:245.

8. Donovan L, Hertel J. A new paradigm for rehabilitation of patients with chronic ankle instability. Phys Sportsmed. 2012 Nov;40(4):41-51. doi: 10.3810/psm.2012.11.1987

9. Konradsen L, Bech L, Ehrenbjerg M, Nickelsen T. Seven years follow-up after ankle inversion trauma. Scand J Med Sci Sports. 2002;12(3):129-135.

10. Evans TA, Hertel J, Sebastianelli WJ. Bilateral postural control deficits after unilateral ankle sprain. Foot Ankle Int. 2004;25:833-9.

11. Delahunt E. Neuromuscular contributions to functional instability of the ankle joint J Bodyw Mov Ther. 2007;11:203-213.

12. Douglas H, Richie Jr. Effects of Foot Orthoses on Patients with Chronic Ankle Instability. J Am Podiatr Med Assoc. 2007;97(1): 19-30.

13. Gregory M. Gutierrez, Thomas W. Kaminski, Al T. Douex. Neuromuscular Control and Ankle Instability. PM R. 2009 April;1:359-365.

14. Holmes A, Delahunt E. Treatment of common deficits associated with chronic ankle instability. Sports Med. 2009;39(3):207-24. doi: 10.2165/00007256-200939030-00003

15. Delahunt E, Monaghan K, Caulfield B. Altered neuromuscular control and ankle joint kinematics during walking in subjects with functional instability of the ankle joint. Am J Sports Med. 2006 Dec;34(12):1970-6.

16. Morrison KE, Hudson DJ, Davis IS, Richards JG, Royer TD, Dierks TA, Kaminski TW. Plantar pressure during running in subjects with chronic ankle instability. Foot Ankle Int. 2010 Nov;31(11):9941000. 10.3113/FAI.2010.0994.

17. Schmidt H, Sauer LD, Lee SY, Saliba S, Hertel J. Increased in-shoe lateral plantar pressures with chronic ankle instability. Foot Ankle Int. 2011 Nov;32(11):1075-80.

18. Huang PY, Lin CF, Kuo LC, Liao JC. Foot pressure and center of pressure in athletes with ankle instability during lateral shuffling and running gait. Scand J Med Sci Sports. 2011 Dec;21(6):461-7. 10.1111/j.1600-0838.2011.01367.x.

19. Roel De Ridder, Tine Willems, Philip Roosen. Plantar pressure distribution during gait and running in subjects with chronic ankle instability. J Foot Ankle Res. 2012;5(Suppl 1):32. 10.3113/FAI.2010.0994.

20. T. Willems, E. Witvrouw, K. Delbaere, A. De Cock, D. De Clercq. Relationship between gait biome- 
chanics and inversion sprains: a prospective study of risk factors. Gait Posture 2005;21:379-387. http:// dx.doi.org/10.1016/j.gaitpost.2004.04.002

21. Mark W. Cornwall, Thomas G. McPoil. Velocity of the Center of Pressure During Walking. J Am Podiatr Med Assoc. 2000;90(7):334-338,

22. Min-Chi Chiu, Hsin-Chieh Wu, Li-Yu Chang. Gait speed and gender effects on center of pressure progression during normal walking. Gait Posture 2013;37:43-48. http://dx.doi.org/10.1016/j.gaitpost.2012.05.030

23. De Cock A, De Clercq D, Willems T, Witvrouw E. Temporal characteristics of foot roll-over during barefoot jogging: reference data for young adults. Gait Posture. 2005;21:432-9. http://dx.doi. org/10.1016/j.gaitpost.2004.05.004

24. P.O. McKeon, M.J. Booi, B. Branam, D.L. Johnson, C.G. Mattacola. Lateral ankle ligament anesthesia significantly alters single limb postural control. Gait Posture. 2010;32:374-377. http://dx.doi. org/10.1016/j.gaitpost.2010.06.016

25. Jay Hertel; W. E. Buckley; Craig R. Denegar. Serial Testing of Postural Control After Acute Lateral Ankle Sprain. J Athl Train. 2001 Oct-Dec;36(4): 363-368.

26. Hertel J, Lauren C. Olmsted-Kramer. Deficits in time-to-boundary measures of postural control with chronic ankle instability. Gait Posture. 2007 Jan;25(1):33-9. http://dx.doi.org/10.1016/j.gaitpost.2005.12.009

27. A. De Cock, J. Vanrenterghem, T. Willems, E. Witvrouw, D. De Clercq. The trajectory of the center of pressure during barefoot running as a potential measure for foot function. Gait Posture. 2008;27:669675. 10.1016/j.gaitpost.2007.08.013

28. Ramachandra P, Maiya AG, Kumar P. Test-retest reliability of the Win-Track platform in analyzing the gait parameters and plantar pressures during barefoot walking in healthy adults. Foot Ankle Spec. 2012 Oct;5(5):306-12.

29. Chiu MC,Wu HC,Chang LY. The Model of Center of Pressure Progression for Adults. Gait Posture. 2013 Jan;37(1):43-8. http://dx.doi.org/10.1016/j.gaitpost.2012.08.010

30. Chiu MC,Wu HC,Chang LY,Wu MH. Center of pressure progression characteristics under the plantar region for elderly adults. Gait Posture. 2013 Mar;37(3):408-12. 10.1016/j.gaitpost.2012.08.010. 


\section{HOJA INFORMATIVA PARA EL PARTICIPANTE}

El propósito de esta información es explicar a los participantes en esta investigación la naturaleza de la misma, así como su rol en el estudio como participantes.

La presente investigación es conducida por D. Miguel Blanco Traba, de la Universidad Rey Juan Carlos. La meta de esta investigación es valorar alteraciones del centro de presión en personas que tuvieron esguince lateral externo de tobillo y compararlo con un grupo sano para ver si hay diferencias biomecánicas en la marcha.

La participación en este estudio es estrictamente voluntaria. La información que se recoja será confidencial y no se usará para ningún otro propósito fuera de los de esta investigación. Se garantizará en todo momento la confidencialidad de los datos de cada uno de los participantes, según la Ley orgánica 15/1999, de 13 de diciembre, de protección de datos de carácter personal, y Real Decreto 1720/2007, de 21 de diciembre, por el que se aprueba el reglamento de desarrollo de la Ley Orgánica 15/1999, de 13 de diciembre, de protección de datos de carácter personal.

Si tiene alguna duda sobre este proyecto, puede hacer preguntas en cualquier momento durante su participación. Igualmente, puede retirarse del estudio en cualquier momento sin que eso le perjudique en forma alguna.

Desde ya le agradecemos su participación. 
Acepto participar voluntariamente en esta investigación, conducida por D. Miguel Blanco Traba. He sido informado de que la meta de este estudio es valorar alteraciones del centro de presión en personas que tuvieron esguince lateral externo de tobillo y compararlo con un grupo sano para ver si hay diferencias biomecánicas en la marcha.

Me han indicado también que tendré que responder preguntas personales de índole deportivo.

Reconozco que la información que yo provea en el curso de esta investigación es estrictamente confidencial y no será usada para ningún otro propósito fuera de los de este estudio sin mi consentimiento. He sido informado de que puedo hacer preguntas sobre el proyecto en cualquier momento y que puedo retirarme del mismo cuando así lo decida, sin que esto acarree perjuicio alguno para mi persona. De tener preguntas sobre mi participación en este estudio, puedo contactar con D. Miguel Blanco Traba en el teléfono 663524661.

Entiendo que una copia de esta ficha de consentimiento se me entregará, y que puedo pedir información sobre los resultados de este estudio cuando éste haya concluido. Para esto, puedo contactar con D. Miguel Blanco Traba en el teléfono anteriormente mencionado. 


\section{ANEXO 2}

CONSENTIMIENTO INFORMADO PARA LA PARTICIPACIÓN EN EL ESTUDIO DE INVESTIGACIÓN:

\section{"Variación de la velocidad del centro de presiones en deportistas con esguince lateral de tobillo"}

- He leído la documentación informativa que se me entregó referente al estudio, la comprendo y estoy de acuerdo en todos sus términos. $\mathrm{He}$ consultado todas mis dudas con los investigadores y considero que he recibido información suficiente sobre la investigación.

- Mi participación es completamente voluntaria y puedo abandonar el estudio en cualquier momento sin tener que dar explicaciones.

- Accedo a que se utilicen los datos obtenidos en el estudio para compartir y/o divulgar en medios de difusión científica, siempre que no se ceda ningún dato de carácter personal que pueda identificarme.

- Presto libremente mi conformidad para la participación en esta investigación.

- En cuanto a los resultados de las pruebas realizadas,

$\square$ Deseo conocer los resultados de mis pruebas.

$\square$ No deseo conocer los resultados de mis pruebas.

El/la participante,

[Firma del/a participante]
El investigador / es,

[Firma del/los investigador/es]
Fdo.:

Fecha:
Fdo.: Miguel Blanco Traba

Fecha: 